\title{
Hyper Rayleigh Scattering of Adenine, Thymine and Cytosine in Neat Water
}

\author{
Christian Jonin*, Estelle Salmon and Pierre-François Brevet \\ Institut Lumière Matière, UMR CNRS 5306, Université Claude Bernard Lyon 1, 10 Rue Ada \\ Byron, F-69622 Villeurbanne cedex, France. \\ * Corresponding author : christian.jonin@univ-lyon1.fr
}

\begin{abstract}
The first hyperpolarizability of the DNA bases Thymine and Cytosine were determined by Hyper Rayleigh Scattering in neat water despite their low solubility. Due to the low intensity levels collected, count statistics was performed instead of the standard dilution procedure. The first hyperpolarizability were found to be $\beta_{\text {Thy }} \quad=(2.99 \pm 0.44) \times 10^{-30}$ esu for Thymine and $\beta_{\text {Cytosine }}=(3.35 \pm 0.21) \times 10^{-30}$ esu for Cytosine. Due to its weak solubility, only an upper limit $\beta_{\text {Adenine }}<(1.82 \pm 0.10) \times 10^{-30}$ esu could be set for Adenine. The first hyperpolarizability of Guanine could not be measured because of its too weak solubility. Theoretical static and $800 \mathrm{~nm}$ dynamic first hyperpolarizability tensor elements were also computed with Gaussian 09 for comparison.
\end{abstract}




\section{INTRODUCTION}

Engineered materials from organic compounds have received a large interest so far in order to develop novel photonic devices for a broad range of applications ${ }^{1-3}$ from sensing to communication devices. In this respect, biomaterials possess an interesting potential, especially in terms of costs, availability and waste. It is therefore attractive to determine the nonlinear optical properties of materials like proteins and $\mathrm{DNA}^{4}$. In the specific case of quadratic nonlinear properties, but this may be extended to cubic properties as well, the nonlinear optical technique of Hyper Rayleigh Scattering (HRS) is an efficient method to determine the first hyperpolarizability of molecules in solution ${ }^{5-7}$. HRS in liquid solutions is based on the incoherent scattering of second harmonic light due to instantaneous molecular orientational fluctuations. The method, in its standard procedure, entails the determination of the second harmonic intensity as a function of the solute molecule concentration. Comparison with a known reference, the so-called external reference method, or the solvent itself, the so-called internal reference method, provides the first hyperpolarizability of the compound and therefore a detailed insight into its electronic structure and dynamics ${ }^{5}$. This method has sparked a large interest in molecular design and has been at the origin of the development of molecular engineering with numerous applications in organic photonics, opto-electronic devices notably ${ }^{5}$. First hyperpolarizabilities of a wide variety of solutes, including neutral and ionic species ${ }^{9-12}$ have thus been measured, in particular for push-pull compounds where donor and acceptor groups provide large first hyperpolarizability magnitudes. Polarization resolved HRS has besides also enabled a direct access to the symmetry of the nonlinear response of these compounds with the determination of the ratio between their dipolar and octupolar character ${ }^{13-}$ ${ }^{16}$. More recently, coherent contributions have been identified in polarization resolved HRS measurements, opening up the possibility to investigate molecular correlation between different nonlinear optical sources like different sites in macromolecules containing more than one 
nonlinear optical chromophore, or different molecules in aggregates or micelles ${ }^{17}$. Studies of biomolecules by HRS have been initiated with the bacteriorhodopsin protein contained in purple membranes and its embedded retinal chromophore ${ }^{18-23}$. Since then, many different proteins have been investigated as they may serve as target probes in tissue imaging for instance ${ }^{24}$.

In this paper, we extend further these previous studies to the experimental determination of the first hyperpolarizability of the DNA bases. The measurements were performed in neat water solutions to avoid any further complexity albeit with the drawback of weak solubilities. Hence, the measurements were performed in a different way as compared to the standard dilution method described above. Here, a single concentration is used but the experiment is performed over long counting times with harmonic photon count statistics analysis. The experimental results are then compared to Gaussian 09 calculations of the static and dynamic first hyperpolarizabilities in order to point to the origin of the nonlinearity.

\section{EXPERIMENTAL SECTION}

HRS Measurements. The experimental HRS setup used in this study has already been described elsewhere ${ }^{25}$. Briefly, the output of a femtosecond Ti:Sapphire laser (Coherent, Chameleon Ultra) with a pulse duration of about $180 \mathrm{fs}$ and an average power of $500 \mathrm{~mW}$ centered at a wavelength of $800 \mathrm{~nm}$ was used to generate incoherent second harmonic scattered light from the sample cell. In order to control the polarization and the energy of the light impinging onto the sample, the beam was passed through a half wave plate followed by a polarizing cube. The fundamental beam was vertically polarized and gently focused using a low NA microscope objective $(\mathrm{X} 16, \mathrm{NA}=0.32)$ onto the sample cell. The latter was made from fused silica and had an optical path length of $5 \mathrm{~mm}$. The focus was nevertheless no more than 
$2 \mathrm{~mm}$ away from the cell walls for alignment convenience. The second harmonic light scattered at right angle at $400 \mathrm{~nm}$ was then sent to a spectrometer (Jobin-Yvon, iHR 320) and a photomultiplier tube (Perkin - Elmer MP 393CL) for spectral cleaning and detection. Each measurement was statistically analyzed and averaged over long time acquisitions and the figures present histograms of these measurements.

Determination of the First Hyperpolarizability. The experimental procedure for the determination of the hyperpolarizability based on unusual statistical counting procedure was the following. Indeed, when the measured HRS intensity is very low, a fine analysis of the output second harmonic intensity must be performed. Usually, it is just a recording for a set time of the harmonic intensity. This operation is repeated after modification of a selected parameter, either concentration, wavelength or input or output polarization angle for example. However, for very low intensities as in the present case, it is necessary to extend the measurement over a much longer time interval. In this case, all parameters of the configuration such as input and output polarization state, fundamental wavelength or solute concentration are fixed. Nevertheless, it is not appropriate to perform this measurement for a single interval of time. It is indeed preferable to repeat this operation over many intervals and extract the counting statistics as a histogram, see Figure 1 where this scheme is applied to neat water. 


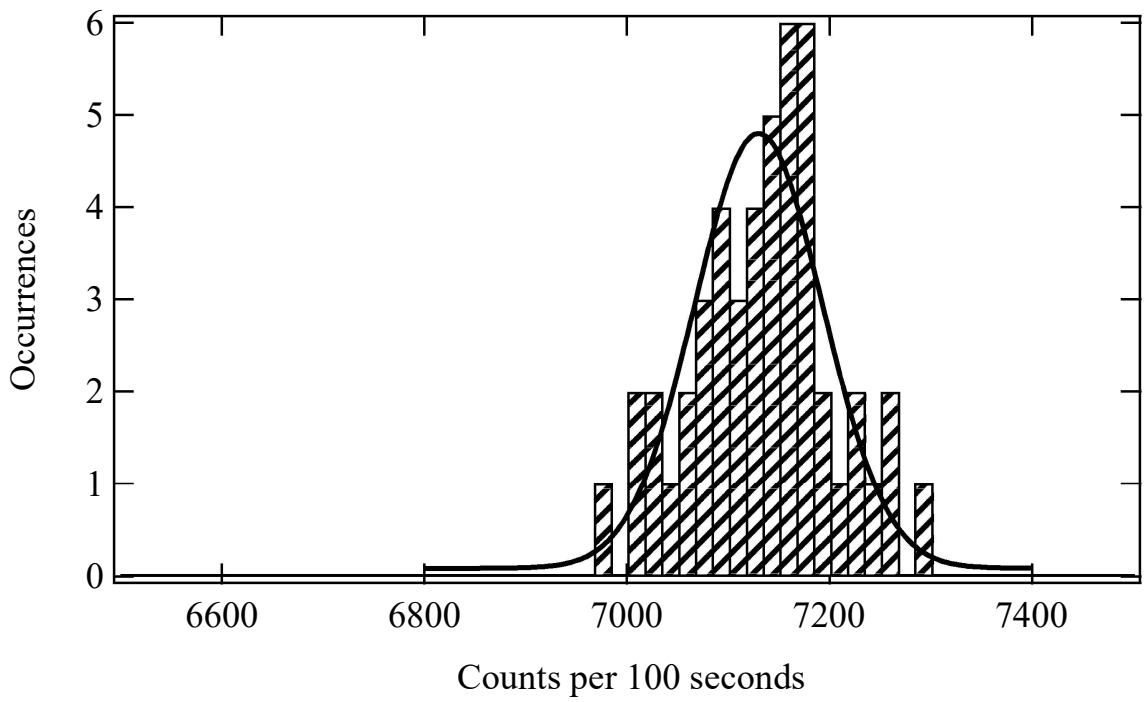

Figure 1: Histogram of the HRS intensity counts per 100 seconds for 47 intervals of equal 300 seconds duration. The full line is a Gaussian fit.

This operation makes it possible to obtain a precise value of the average count intensity at the set harmonic wavelength as well as its standard deviation. In addition, as can be seen in Figure 1 , the counting distribution provides information on the process being studied. In the present case, we note that the counting statistics obeys a Gaussian law as expected for a large number of repeated independent measurements. We can therefore determine the mean value and the standard deviation, in the present case for neat water 7130 counts per 100 seconds with a standard deviation of 89.2 counts per 100 seconds. These parameters will allow to clearly determine the first hyperpolarizabilities along with their error and to carry out finer comparisons than with simple measurements over shorter times where the count statistics is poorer. In the dilution procedure, only two sample concentrations were then used due to the weak solubility of the DNA bases in neat water and thus the difficulty to prepare a set of well-defined sample cells of different concentrations. For each DNA bases, the measured HRS intensity was normalized with respect to the square of the incident power to account for temporal fluctuations in the laser power. The count statistics for neat water and the DNA bases performed in the same 
operating conditions were both adjusted with a Gaussian function. The first hyperpolarizability was then determined with respect to that of neat water knowing the water and DNA base number densities. The first hyperpolarizability of water was taken from a previous work as $0.087 \times 10^{-30} \mathrm{esu}^{26}$.

Chemicals. Water was purified by reverse osmosis followed by ion exchange (Millipore, Milli-Q SP reagent system). Adenine (Sigma-Aldrich, 99\% purity), Cytosine (Sigma-Aldrich, 99\% purity), Guanine (Sigma-Aldrich, 99\% purity) and Thymine (Sigma-Aldrich, 99\% purity) were used as received. Solubility values of the different DNA bases are $1.03 \mathrm{mg} / \mathrm{ml}$ for Adenine, $8 \mathrm{mg} / \mathrm{ml}$ for Cytosine and $10.8 \mathrm{mg} / \mathrm{ml}$ for Thymine whereas Guanine is insoluble in water ${ }^{24-26}$. Aqueous solutions of $5 \mathrm{mM}$ Adenine, 5 and $10 \mathrm{mM}$ Thymine and 5 and $10 \mathrm{mM}$ Cytosine were prepared. No solution from Guanine could be prepared due to its too large insolubility. Figure 2 presents the four DNA bases chemical formula. The frame is indicated in the standard orientation to allow us to compare our results with the literature ones ${ }^{30}$. 


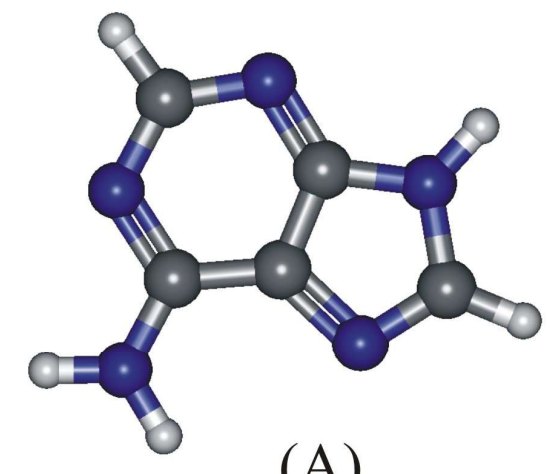

(A)

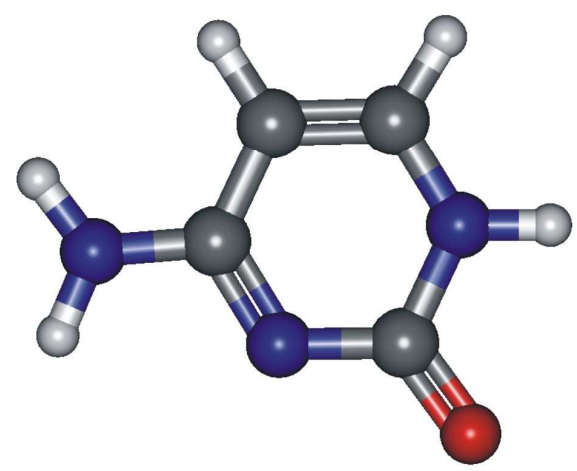

(C)

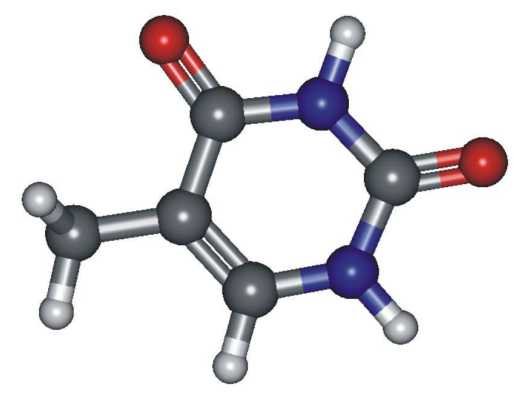

(T)
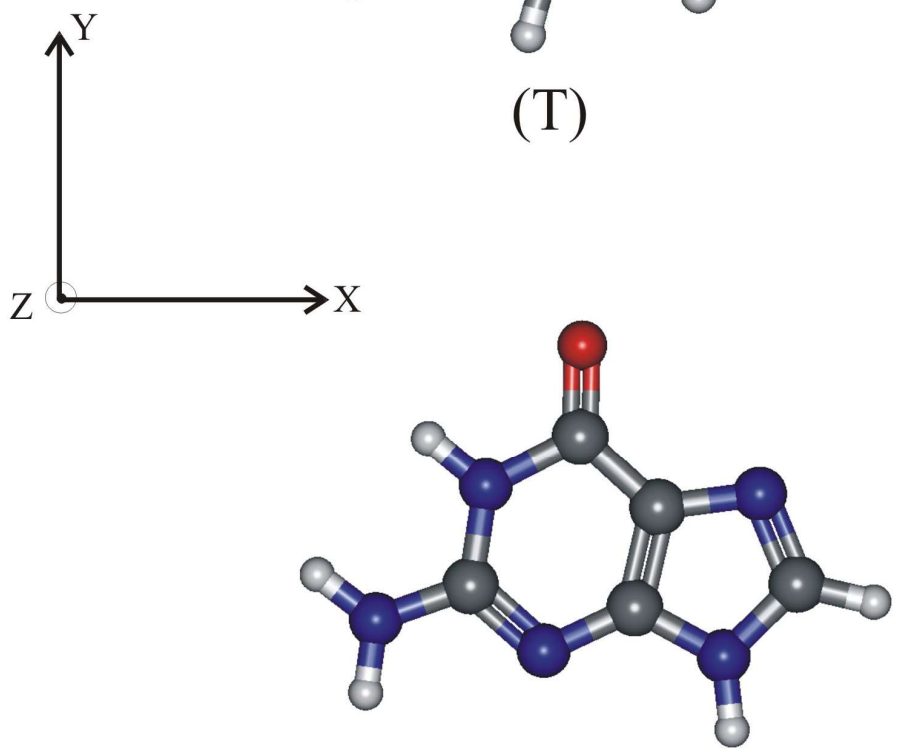

(G)

Figure 2: Adenine (A), Thymine (T), Cytosine (C) and Guanine (G) formula with the frame axes as used in the theoretical calculations. White balls represent $\mathrm{H}$ atom, red balls Oxygen atoms, blue balls nitrogen atoms and grey balls Carbon atoms ${ }^{28}$.

\section{RESULTS AND DISCUSSION}

Experimental determination of the first hyperpolarizabilities. Due to the relatively low solubility of all DNA bases in neat water, only low concentration aqueous solutions could be prepared. As a result, and considering the chemical formula of each DNA bases and the 
potential hyperpolarizabilities expected, the resulting HRS intensities could not be distinguished from that recorded for neat water, see Figure 3, using short counting times.

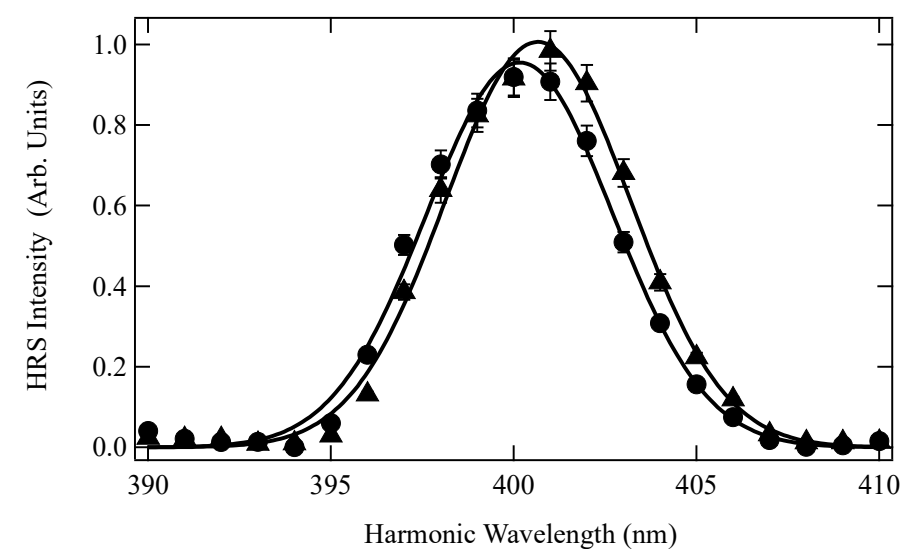

Figure 3: Neat Water (triangles) and a $10 \mathrm{mM}$ Thymine aqueous solution (disks) HRS line for a $800 \mathrm{~nm}$ fundamental excitation. Corresponding Gaussian fits are provided as full lines. Neat water HRS intensity maximum is normalized to unity.

Standard deviations obtained from single short acquisitions were too large to unambiguously separate the contribution of the DNA base itself from that of neat water. Hence, longer acquisition times were used along with a statistical approach entailing repeated measurements in order to increase the sensitivity. Typically, in the case of Thymine for example, the HRS intensity counts were recorded during 14400 seconds split into 48 time intervals of 300 seconds duration each for both neat water and the Thymine aqueous solution, see Figure 4. Plots reported in Figure 3 were nevertheless required initially to ensure that no spurious light like multiphoton excited fluorescence contribute to the detected intensity. 


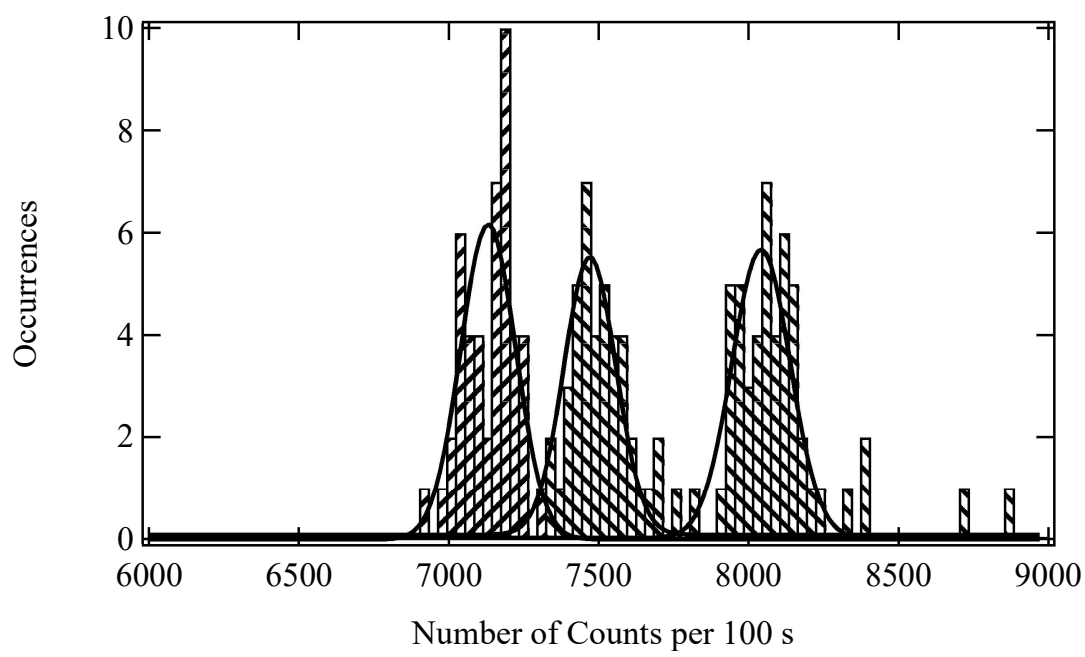

Figure 4(a): Count distributions obtained for the HRS intensity for (left) neat water, (Center) $5 \mathrm{mM}$ and (Right) $10 \mathrm{mM}$ Thymine aqueous solutions. See text for the experimental conditions.

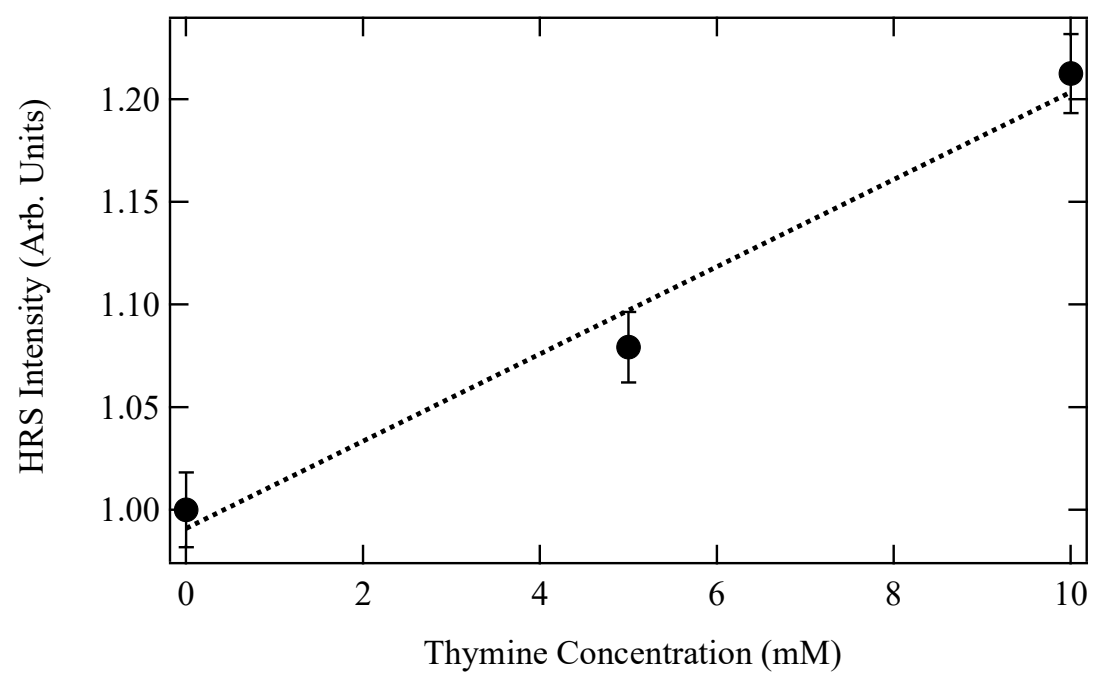

Figure 4(b): Average HRS intensity as a function of Thymine Concentration. Error bars correspond to standard deviation of the adjustment procedure.

These histograms, constructed at the harmonic frequency are thus the sum of the background noise and the HRS intensity for neat water whereas they are the sum of the background noise, to which is added the contribution of water and Thymine for the Thymine solutions. From the 
recorded HRS spectra presented on Figure 3, it is observed that no multiphoton excited fluorescence occurs indeed and the background noise was subtracted using the HRS line profile. With the Gaussian adjustments made on Figure 4(a), the amplitude of each Gaussian line is determined, along with the standard deviation. In the case of neat water for example, the average value is 7131 counts per 100 seconds for a standard deviation of 130 counts leading to $71.31 \pm 1.30$ counts/s. For the $10 \mathrm{mM}$ Thymine solution, the average value is 8041 counts per 100 seconds with a standard deviation of 138 counts leading to $80.41 \pm 1.38$ counts/s. Then, using the equation the HRS intensity as a function of the hyperpolarizability as follows:

$$
I_{H R S}=G\left(N_{\text {Water }}\left\langle\beta_{\text {Water }}^{2}\right\rangle+N\left\langle\beta^{2}\right\rangle\right) I^{2}
$$

and the internal reference method, the square of the first hyperpolarizability of Thymine is obtained from the slope of Figure 4(b) :

$$
I_{H R S} \propto 1+N_{\text {Thymine }} \frac{\left\langle\beta_{\text {Thymine }}^{2}\right\rangle}{N_{\text {Water }}\left\langle\beta_{\text {Water }}^{2}\right\rangle}
$$

Using $\quad \beta_{\text {water }}=\sqrt{\left\langle\beta_{\text {water }}{ }^{2}\right\rangle}=0.087 \pm 0.009 \times 10^{-30} e s u^{23}$ for the neat water hyperpolarizability, we obtain for the Thymine first hyperpolarizabilty $\beta_{\text {Thy }}=$ $\sqrt{\left\langle\beta_{\text {Thymi }}{ }^{2}\right\rangle}=(2.99 \pm 0.44) \times 10^{-30}$ esu. This value is rather weak but can be compared to aromatic and non aromatic amino acid first hyperpolarizabilities for example. Hyperpolarizabilities values of $4.7 \times 10^{-30} \mathrm{esu}, 4.1 \times 10^{-30}$ esu and $0.3 \times 10^{-30}$ esu have been reported for Tryptophane, Tyrosine and Glycine respectively ${ }^{26}$. In particular, it appears that the Thymine first hyperpolarizability is within the range of those of Tryptophane and Tyrosine, two aromatic amino acids whereas that of Glycine is about ten times smaller, as 
expected for a non aromatic molecule. The same experimental procedure was carried out with the other DNA bases. However, because Guanine is not soluble in water, we were not able to present results for Guanine and therefore could not extract a first hyperpolarizability.

The procedure was thus repeated for the case of Cytosine. First, a short spectrum of the HRS line was recorded to ensure the absence of multiphoton excited fluorescence in the $400 \mathrm{~nm}$ signals recorded (Figure 5) and then long counting times were used to measure the HRS intensities for two different concentrations, see Figure 6(a): Count distributions obtained for the HRS intensity for (left) neat water, (Center) $5 \mathrm{mM}$ and (Right) $10 \mathrm{mM}$ Cytosine aqueous solutions.

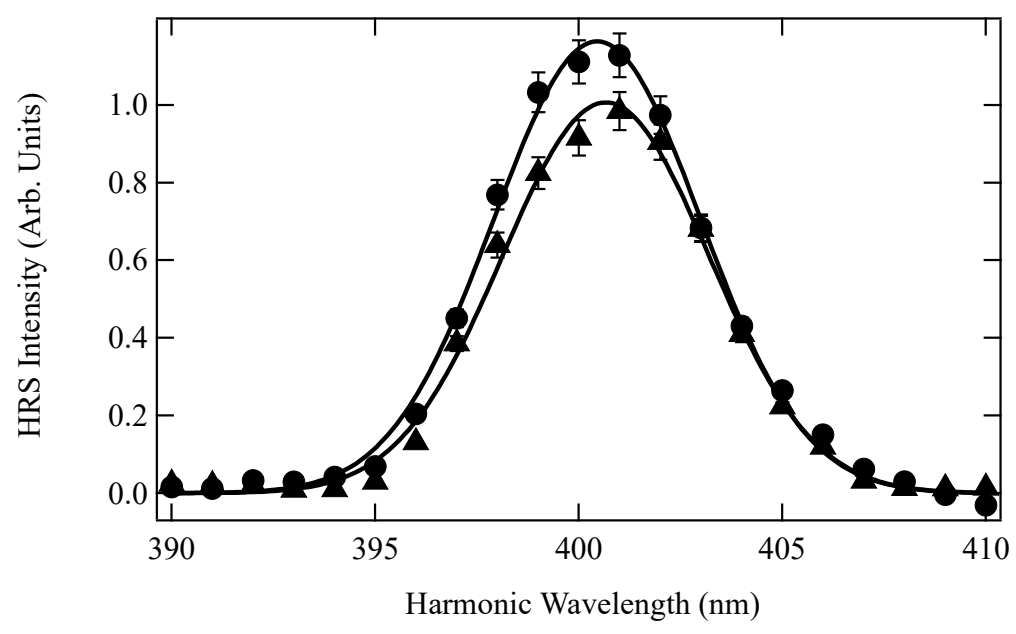

Figure 5: Neat Water (full triangles) and a $10 \mathrm{mM}$ Cytosine aqueous solution (disks) HRS line for a 800 $\mathrm{nm}$ fundamental excitation. Corresponding Gaussian fits are provided as full lines. Neat water HRS intensity maximum is normalized to unity. 


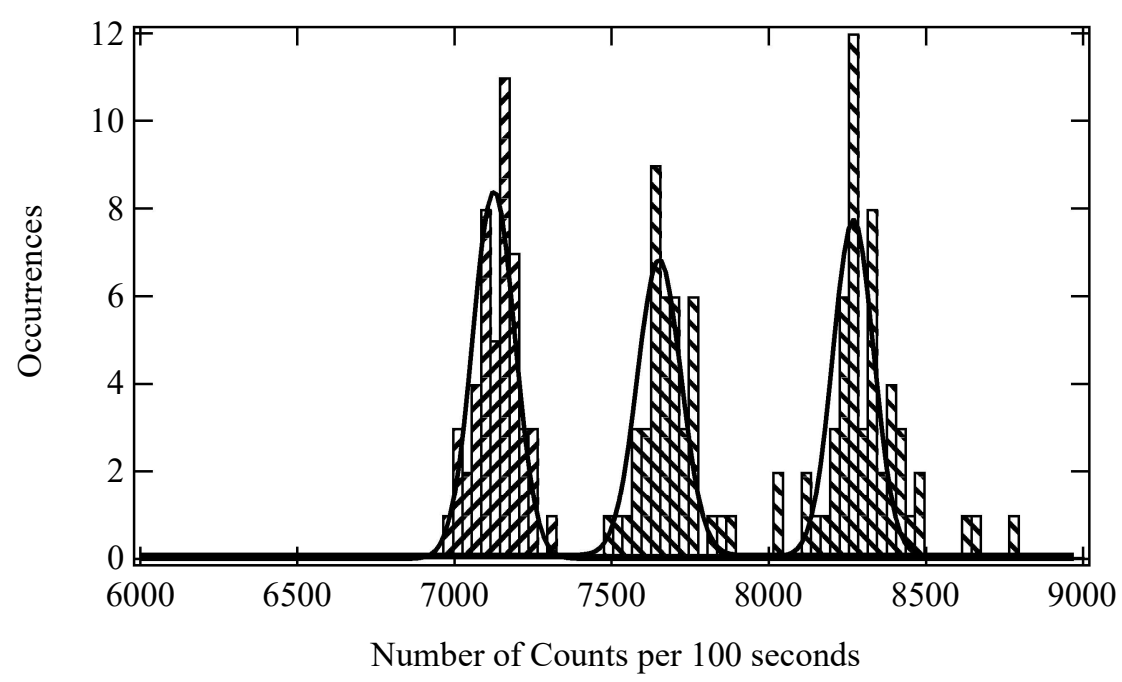

Figure 6(a): Count distributions obtained for the HRS intensity for (left) neat water, (Center) $5 \mathrm{mM}$ and (Right) $10 \mathrm{mM}$ Cytosine aqueous solutions.

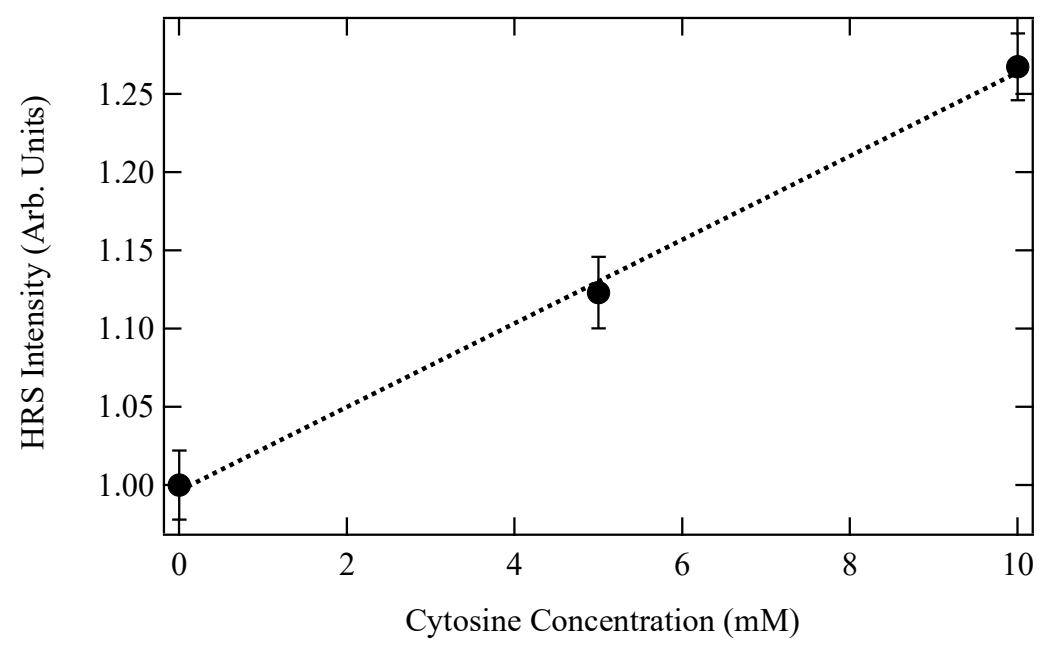

Figure 6(b): Average HRS intensity as a function of Cytosine Concentration. Error bars correspond to standard deviation of the counting procedure.

The Gaussian fits yielded the average value for neat water of $71.24 \pm 0.93$ counts/s, $76.50 \pm$ 0.62 counts $/ \mathrm{s}$ for the $5 \mathrm{mM}$ Cytosine solution and $82.69 \pm 0.69$ counts $/ \mathrm{s}$ for the $10 \mathrm{mM}$ Cytosine solution. Using the neat water hyperpolarizability for reference and Eq.(2) adapted for 
Cytosine, the value of the first hyperpolarizability of Cytosine is found to be $\beta_{\text {Cytosine }}=$ $\sqrt{\left\langle\beta_{\text {Cytosine }}{ }^{2}\right\rangle}=(3.35 \pm 0.21) \times 10^{-30}$ esu.

Finally, in the case of Adenine, the HRS line spectrum exhibits for Adenine a rather strong multiphoton excited fluorescence background that must be subtracted before getting the first hyperpolarizability, see Figure 7.

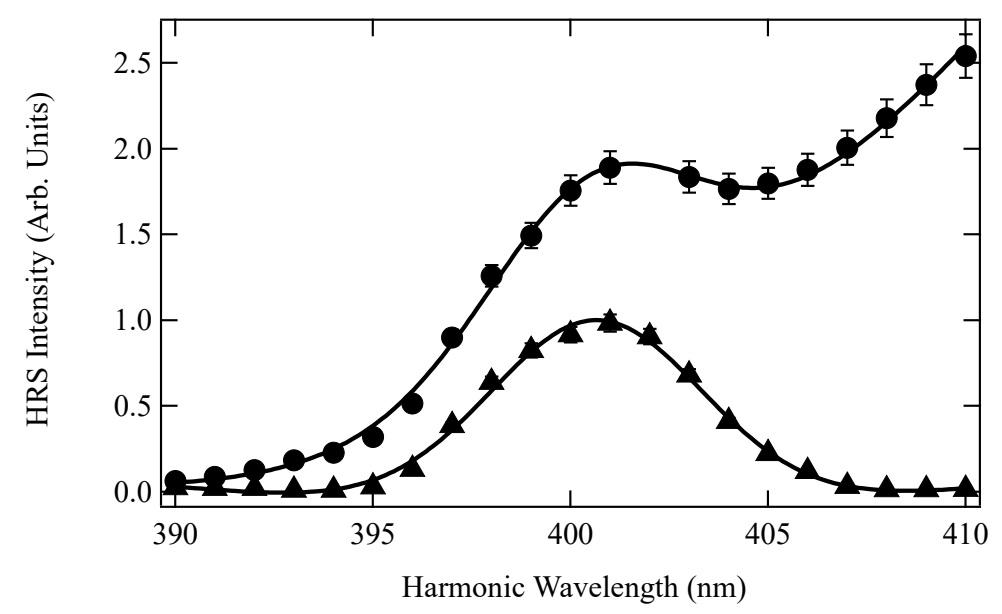

Figure 7: Neat Water (triangles) and a $5 \mathrm{mM}$ Adenine aqueous solution (disks) HRS line for a $800 \mathrm{~nm}$ fundamental excitation. Corresponding Gaussian fits are provided as full lines. Neat water HRS intensity maximum is normalized to unity.

From the data plotted in Figure 7, we were nevertheless able to extract the height of the HRS signal coming from the Adenine by fitting the data with a Gaussian function superposed to a polynomial function of second order. No spurious signal counts were collected from the neat water solution, as previously seen, apart from the noise background that can be subtracted. Then, count histograms were performed as well following the above procedure, see Figure 8. 


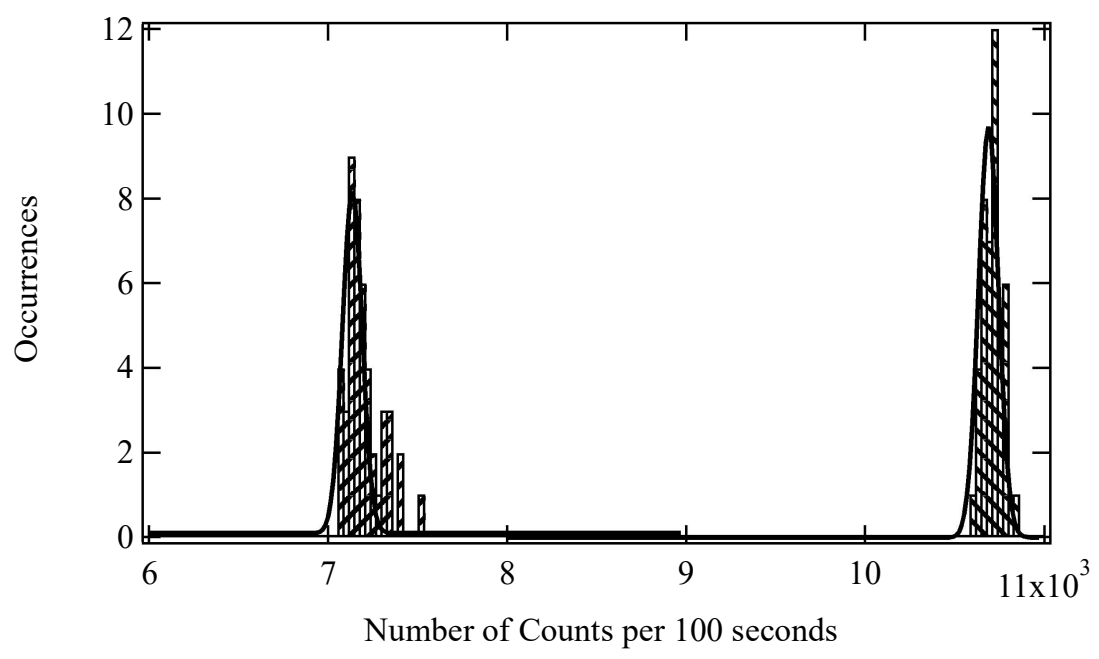

Figure 8: Count distributions obtained for (left) neat water and (right) $5 \mathrm{mM}$ Adenine aqueous solution.

In the histograms, the intensity measured for the neat water solution again corresponds to the noise background augmented with the neat water HRS intensity counts. For the Adenine aqueous solution, the intensity corresponds to the noise background augmented with the neat water contribution and the Adenine contribution, both the multiphoton excited fluorescence and the HRS counts. The noise background contribution was again determined by inspection at 390 $\mathrm{nm}$, slightly off the HRS line. However, in the case of Adenine, the multiphoton excited fluorescence contribution was found to be an overwhelming contribution as compared to the HRS contribution precluding the use of the above described procedure. It appears indeed that from the data reported in Figure 7, the HRS intensity is identical for neat water and the Adenine solution. Therefore, no first hyperpolarizability could be determined as a result of the combined limited solubility and weak HRS response. However, an estimate of the first hyperpolarizability for Adenine can nevertheless been made. Considering the standard deviation of the histogram of neat water, we may argue that Adenine HRS intensity could be detected provided its total signal including background noise and HRS intensity of neat water and Adenine itself equals the total signal of water augmented by two standard deviations of the neat water signal. If this were indeed observed, Adenine would be indeed observed from the experimental data. With 
this procedure, and using as a superior limit for the standard deviation de square root of the number of counts per 100 seconds, that is including the noise background and HRS intensity for neat water, we determine a value less than $(1.82 \pm 0.1) \times 10^{-30}$ esu for Adenine first hyperpolarizability. This value is thus an estimated upper limit.

From these measurements, all these experimental results lead to the following ordering of the first hyperpolarizability of the three Cytosine, Thymine and Adenine, namely $\beta_{\text {cytosine }}>$ $\beta_{\text {Thymi }}>\beta_{\text {Adenine }}$ and no value is available unfortunately for Guanine due to its too weak solubility.

Theoretical calculation of the first hyperpolarizabilities. In order to get a deeper insight into the experimental results and the origin of the first hyperpolarizabilities of the DNA bases, a simple theoretical calculation was performed for the first hyperpolarizabilities with Gaussian 09 in vacuum. For each base, the geometrical structure was first optimized using the DFT exchange correlation functional B3LYP in Gaussian 09. Atom-centered split valence with polarization functions $6-31+\mathrm{G}^{*}$ for $\mathrm{C}, \mathrm{H}, \mathrm{O}$ and $\mathrm{N}$ were used. The static and dynamic first hyperpolarizabilities were obtained at the Hartree-Fock (HF) level. The first hyperpolarizability is a third rank tensor involving 27 elements, a number that can be reduced using the intrinsic frequency doubling symmetry, i.e. permutation of the last two indices. In the static case, the Kleinman symmetry may be also used, i.e. full permutation of the indices, reducing this number of elements to 10 only. The static first hyperpolarizabilities of the four DNA bases are reported in Table 1.

Table 1: static $\beta_{i j k}$ components and $\beta$ calculated at HF level using the $6-31+G^{*}$ basis set with Gaussian 09 for the four DNA bases. All values reported in atomic units. Conversion factors 
are from atomic units to esu 1 a.u. $=8.639 \times 10^{-33} \mathrm{esu}$ and from atomic units to SI units 1 a.u. $=3.206361 \times 10^{-53} \mathrm{C}^{3} \mathrm{~m}^{3} \mathrm{~J}^{-2}$.

\begin{tabular}{lcccccccccc}
\hline & $\beta_{x x x}$ & $\beta_{x x y}$ & $\beta_{y x y}$ & $\beta_{y y y}$ & $\beta_{x x z}$ & $\beta_{y x z}$ & $\beta_{y y z}$ & $\beta_{z x z}$ & $\beta_{z y z}$ & $\beta_{z z z}$ \\
\hline A & -18.3 & 24.5 & 54.5 & 81.5 & -0.02 & 0.003 & -0.02 & 1.55 & -13 & -0.02 \\
G & 98.5 & 45.4 & -23.8 & -22.45 & -0.06 & 0.03 & 0.005 & 6.6 & -15.7 & -0.025 \\
$\mathrm{~T}$ & -54 & -9.5 & 47.8 & 12.1 & -0.004 & 0 & 0.005 & -10.15 & -8.15 & -0.004 \\
$\mathrm{C}$ & 125 & 25.9 & -79.5 & 34.3 & -0.07 & -0.01 & 0.03 & -28.95 & 32.05 & -0.001
\end{tabular}

To provide further insights into the comparison between theoretical and experimental values, dynamic first hyperpolarizabilities were also evaluated at the experimental fundamental excitation wavelength of $800 \mathrm{~nm}(\hbar \omega=0.057$ a.u. $)$. In this case, Kleinman symmetry breaks down and 18 elements are calculated. The results are presented in Table 2.

Table 2: Dynamic $\beta_{\mathrm{ijk}}$ components and $\beta$ calculated at HF level using the $6-31+\mathrm{G}^{*}$ basis set with Gaussian 09 for the four DNA bases. All values are reported in atomic units. Conversion factors are from atomic units to esu 1 a.u. $=8.639 \times 10^{-3}$ esu and from atomic units to SI units 1 a.u. $=3.206361 \times 10^{-53} \mathrm{C}^{3} \mathrm{~m}^{3} \mathrm{~J}^{-2}$.

\begin{tabular}{ccccc}
\hline $\begin{array}{c}\text { Tensor } \\
\text { components }\end{array}$ & Adenine & Guanine & Thymine & Cytosine \\
\hline$\beta_{x x x}$ & 24.45 & 12.9 & -56.5 & 162 \\
\hline$\beta_{y x x}$ & 43.2 & 21 & -6.85 & 10.05 \\
\hline$\beta_{z x x}$ & -0.035 & -0.105 & 51.5 & -0.095 \\
\hline
\end{tabular}




\begin{tabular}{|c|c|c|c|c|}
\hline$\beta_{x y x}$ & 49.85 & 18.95 & -0.0045 & 30.05 \\
\hline$\beta_{y y x}$ & 69.4 & -27.8 & 0.001 & -107.5 \\
\hline$\beta_{z y x}$ & 0.005 & 0.045 & -11.1 & -0.12 \\
\hline$\beta_{x y y}$ & 60.75 & -17.95 & -8.65 & -106 \\
\hline$\beta_{y y y}$ & 111.8 & 15.3 & 51 & 40.3 \\
\hline$\beta_{z y y}$ & -0.025 & 0.01 & 16.5 & 0.04 \\
\hline$\beta_{x z x}$ & -0.045 & -0.135 & 0.0005 & -0.095 \\
\hline$\beta_{y z x}$ & 0.005 & 0.05 & 0.005 & -0.005 \\
\hline$\beta_{z z x}$ & 2.7 & 5.55 & -8.85 & -40.3 \\
\hline$\beta_{x z y}$ & 0.01 & 0.06 & -0.0045 & -0.01 \\
\hline$\beta_{y z y}$ & -0.025 & 0.01 & 0.001 & 0.04 \\
\hline$\beta_{z z y}$ & -20.95 & -22.4 & 0.0005 & 46.25 \\
\hline$\beta_{x z z}$ & 3.85 & 12.165 & -11.1 & -40.2 \\
\hline$\beta_{y z z}$ & -18.15 & -25.65 & -9.4 & 47.8 \\
\hline$\beta_{z z z}$ & -0.025 & -0.035 & -0.0045 & -0.004 \\
\hline
\end{tabular}

In this calculation, the reference frame as given in Figure 1 is used where the $\mathrm{z}$ axis is normal to the aromatic cycles. The magnitude of the elements of the first hyperpolarizability tensor for Adenine, Thymine and Cytosine are found on average much larger than that of Guanine. Besides, the values obtained for Guanine are in very good agreement of those calculated by Alparone $^{30}$. In order to perform a better comparison with the experimental data, the Hyper Rayleigh Scattering molecular first hyperpolarizabilty in the laboratory frame was computed from the above microscopic first hyperpolarizability tensor elements using : 


$$
\left\langle\beta_{H R S}\right\rangle=\sqrt{\left\langle\beta_{Z Z Z}^{2}\right\rangle^{2}+\left\langle\beta_{X Z Z}^{2}\right\rangle}
$$

where the $\mathrm{X}$ direction is assumed to be the fundamental light beam propagation whereas the unpolarized output harmonic light is collected in the Y-direction. The elements $\left\langle\beta_{Z Z Z}^{2}\right\rangle$ and $\left\langle\beta_{X Z Z}^{2}\right\rangle$ are the macroscopic averages calculated from the first hyperpolarizability microscopic components $\beta_{i j k}$ given in Table 2 using the standard formulae between the molecular averages and the molecular first-order hyperpolarizability tensor components ${ }^{31}$.

The ordering of the calculated HRS molecular first hyperpolarizability for the four bases is then

$$
\begin{gathered}
\beta_{\text {Cytosine }}\left(0.43 \times 10^{-3} \text { esu }\right)>\beta_{\text {Adenine }}\left(0.32 \times 10^{-30} \text { esu }\right)>\beta_{\text {Guanine }}(0.17 \times \\
\left.10^{-30} \text { esu }\right)>\beta_{\text {Thymine }}\left(0.08 \times 10^{-30} \text { esu }\right) .
\end{gathered}
$$

Cytosine therefore displays the largest first hyperpolarizability, both experimentally and theoretically. On the opposite, we observe inversion for that of Adenine and Thymine. It must be pointed however that the solvent medium is not taken into account for the theoretical first hyperpolarizabilities and may be the cause of the difference between the experimental and the theoretical data. In order to discuss further the first hyperpolarizability values, we also provide the dipole moments obtained using B3LYP/6-31+ $\mathrm{G}^{*}$ level for the four bases. The ordering of the theoretical dipole moment magnitudes $\mu$ is found to be:

$$
\mu_{\text {Guanine }}(7.253 D)>\mu_{\text {cytosine }}(7.09 D)>\mu_{\text {Thymine }}(4.70 D)>\mu_{\text {Adenine }}(2.46 D) \text {. }
$$

The link between the electric dipole moments of an organic molecule having donor -acceptor subsituent and the first order hyperpolarizability has been recognized in the literature ${ }^{32}$. Large 
first hyperpolarizabilities result from an intramolecular charge transfer due to the $\pi$-conjugated electron framework disturbed by the presence of electron donor and acceptor groups.

This argument can be shown with a natural population analysis ${ }^{33-34}$ for the four bases, see Figure 9.

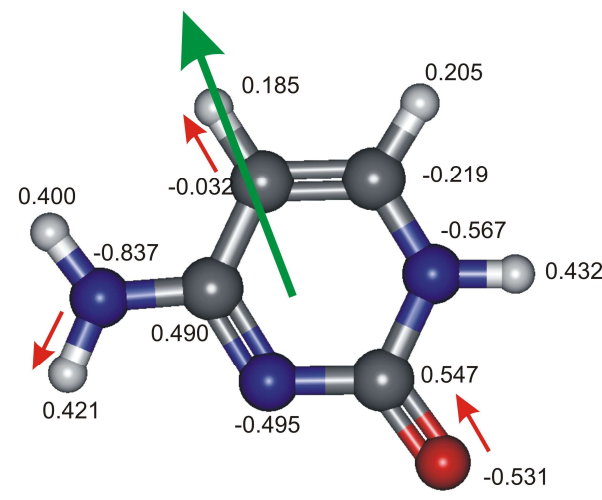

(a)

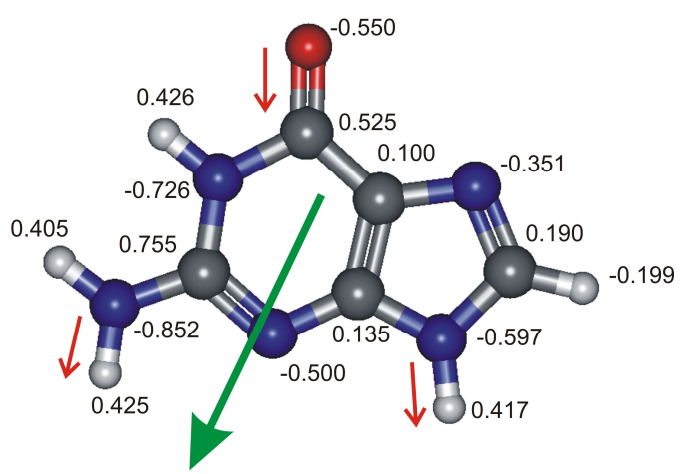

(b)
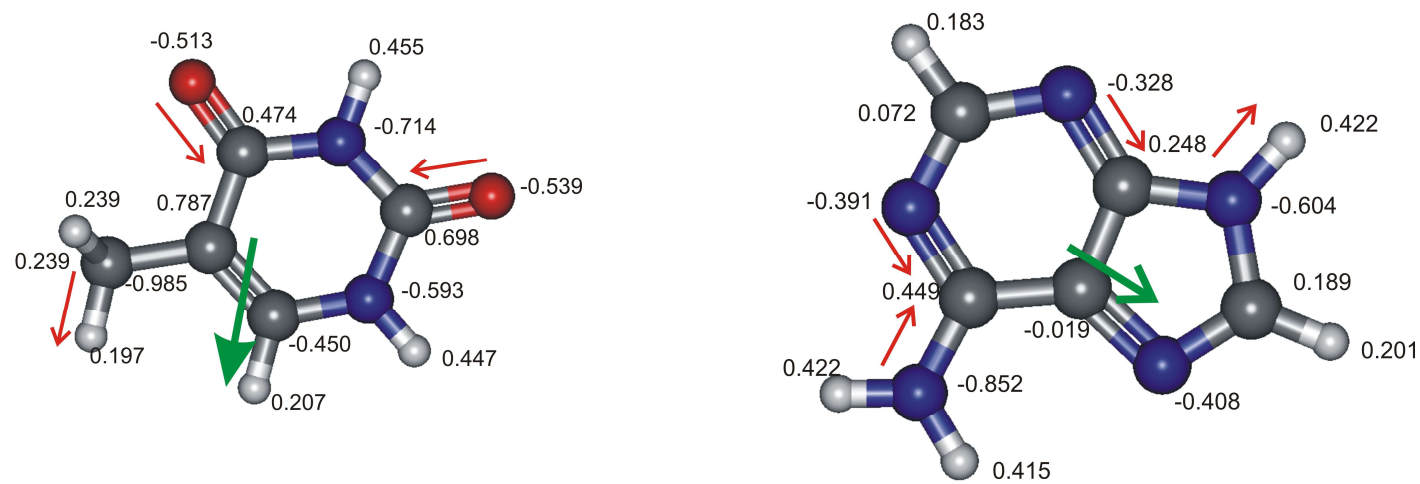

(c)

Figure 9 : Dipole moments (green arrow) and NPA atomic charges of the Cytosine (a), Guanine (b), Thymine (c) and Adenine (d). Color code: white hydrogen, gray carbon, red oxygen, blue nitrogen. Red arrows indicate polar bonds orientation. 
The Figure (a) displays the green vectorial representation of the dipole moment for Cytosine. For this compound, polar bonds are parallel reinforcing the donor - acceptor scheme and resulting in a relatively important dipole moment $\mu$ compared with the others compounds. However, in the case of Adenine, the bond moment, see the red arrows in Figure 9(d), are in mutual opposition. Consequently the dipolar contributions tend to cancel each other out leading to the lowest value dipole moment. The Guanine dipole moment is for the same reason as that of Cytosine fairly enhanced whereas for Thymine the bond moments are essentially in mutual opposition. Thus, Cytosine and Thymine present similar experimental first hyperpolarizability values despite the presence of a single aromatic cycle, at odds with the theoretical case. However, the charge transfer as well as the meta position of the electron donor group $\mathrm{NH}_{2}$ and the electron acceptor group $\mathrm{C}=\mathrm{O}$ must be considered in determining the nonlinearity. It must however be pointed out that in water, DNA bases have the ability to be present under several tautomeric structures. These tautomers may possess differing nonlinear optical properties, a feature not accounted for in the present situation. Guanine was also studied theoretically in the past and was exhibiting such a behavior ${ }^{30}$. Nevertheless, the present findings call for an indepth investigation of the first hyperpolarizabilities of DNA bases with careful account of the different tautomeric structures and the solvent effect.

\section{CONCLUSIONS}

The experimental first hyperpolarizability of Thymine and Cytosine DNA bases dispersed in neat water were reported as measured by HRS. For Adenine, only an upper limit can be provided due to the presence of a large fluorescence background whereas for Guanine no value is reported due to its too low solubility. Interestingly, Thymine and Cytosine first hyperpolarizability values are rather similar. This result is somewhat at odds with the theoretical values reported using Gaussian 09 at the HF level in vacuum, possibly due to the lack of the 
account of the solvent. Thymine shows a much smaller first hyperpolarizability as compared to Cytosine despite a larger delocalized electronic structure. It is suggested that the role of the tautomeric structures and the solvent cannot be neglected and must be incorporated in the calculations for a better agreement with the experimental values reported.

\section{SUPPLEMENTARY MATERIAL}

In Supplementary Information file, we have added in Tables S1 and S2 the static and the dynamic hyperpolarizability tensor values within the dipole oriented reference frame, namely with the z-axis along the dipole moment allowing to evaluate influence of the dipole moment orientation on the first hyperpolarizability tensor elements whereas the y axis is pointing up, and the $\mathrm{x}$ axis completes the right-handed coordinate system.

\section{AUTHORS CONTRIBUTIONS}

All authors contributed equally to this work.

\section{ACKNOWLEDGMENTS}

Centre National de la Recherche Scientifique (CNRS) and Université Claude Bernard, LYON 1 are acknowledged. There are no conflicts to declare

\section{DATA AVAILABILITY}

The data that support the findings of the study are available from the corresponding author upon reasonable request. 


\section{REFERENCES}

1. F. Ouchen, E. Gomez, D. Joyce, A. Williams, S. Kim, E. Heckman, L. Johnson, P. Yaney, 6, N. Venkat, A. StecK1, F. Kajzar, I. Rau, A. Pawlicka, P. Prasad and J. Grote, Nonlinear Optics and Quantum Optics, 46, pp. 199-225(2014)

2. O. Krupka, A. El-Ghayoury, I. Rau, B. Sahraoui, J.G. Grote, F. Kajzar, NLO properties of functionalized DNA thin films, Thin Solid Films, 516, 8932-8936 (2006)

3. J.G. Grote, D.E Diggs, R.L. Nelson, J.S. Zetts, F.K. Hopkins, N. Ogata, J.A. Hagen, E. Heckman, P.P. Yaney, M.O. Stone, L.R. Dalton, DNA photonics, Mol. Cryst. Liq. Cryst., 426, 3-17 (2005)

4. D. Chevizovich, D. Michieletto, A. Mvogo, F. Zakiryanov, and S. Zdravkovic, R. Soc. Open Sci, 7, 200774(2020).

5. K. Clays, A. Persoons, Phys. Rev. Lett., 66, 2980-2983(1991).

6. V. Rodriguez, J. Phys. Chem. C, 121, 8510-8514(2017).

7. A. Rodriguez, M. Soljačić, J. D. Joannopoulos, and S. G. Johnson, Optics Express, 15, 73037318(2007).

8. J. Zyss, I. Ledoux, Chem. Rev., 94, 77-105(1994).

9. G. Heesing, A. G. T. Ruiter,N. F. Van Hulst,B. Bolger, Phys.Rev.Lett, 71, 999-1002(1993).

10. P. Kaatz, D. P. Shelton, Rev. Sci. Instr., 67, 1438-1444(1996).

11. T. Verbiest, SPIE Washington, 1775, 206-212(1992).

12. T. W. Chui,K. Y. Wong, J. Chem. Phys, 109, 1391-1396(1998)

13. K. Clays, and A. Persoons, Rev. Sci. Instrum., 65, 2190-2194(1994)

14. S. Stadler, G. bourhill, and C. Brauche,J. Chem. Phys., 100, 292-296(1995).

15. M. Blanchard-Desce et al. 1996, Ed. Elsevier: Oxford, 10, 833-863.

16. A. Sen,P. C. Ray, P. K. Das, and V. Krishnan, J. Phys. Chem., 100, 19611-19613(1996).

17. G. Revillod, J. Duboisset, I. Russier-Antoine, E. Benichou, C. Jonin, and P-F.Brevet, Symmetry, 13, 206(2021).

18. K. Clays, E. Hendrickx, M. Triest, T. Verbiest, A. Persoons, C. Dehu, J-L. Brédas, Science, 262, 1419-1422(1993).

19. P.K Schmidt, and G. W. Rayfield, Appl. Opt., 33, 4286-4292(1994).

20. E. Hendrickx,A. Vinckier, K. Clays, A. Persoons, J. Phys. Chem., 100, 19672-19680(1996).

21. E. Hendrickx, K. Clays, A. Persoons, C. Dehu, J-L. Brédas, J. Am. Chem. Soc., 117, 3547-3555(1995).

22. K. Clays, S. van Elshocht, and A. Persoons, Opt. Lett., 25, 1391-1393(2000).

23. K. Clays, S. van Elshocht, M. Chi,E. Lepoudre, A. Persoons, J. Opt. Soc. Am. B, 18, 14741482(2001).

24. Jonin, C, Ray, C et Salmon, E., Leclerc. P, Montcel. B, Mahieu-Williame, L., Brevet, PF., J. P. P. A, 402, 112812(2020). 
25. G. Revillod, J. Duboisset, I. Russier-Antoine, E. Benichou, G. Bachelier, C. Jonin, and P.F. Brevet, J. Phys. Chem. C, 112, 2716(2008).

26. J. Duboisset, A. Deniset-Besseau, E. Benichou, I. Russier-Antoine, N. Lascoux, C. Jonin, F. Hache, M. C. Schanne-Klein, and P. F. Brevet, J. Phys. Chem. B, 117, 9877-9881(2013).

27. S.H. Yalkowsky, and R. M. Dannenfelser, 5th ed. School of Pharmacy, University of Arizona(1990).

28. S. H. Yalkowsky, and S. Bannerjee, Dekker, M, New York(1992).

29. S. H. Yalkowsky, and S. Bannerjee, water research, 30, 2222-2225(1996).

30. A. Alparone, Journal of Molecular Modeling, 19, 8(2013).

31 R. Bersohn, Y. H. Pao and H. Frisch, J. Chem. Phys., 45,3184 - 3198 (1966).

32. V. Verdolino, R. Cammi, B. H. Munk, and H. B. Schlegel, J. Phys. Chem. B, 112, 1686016873(2008).

33. S. Arulmozhi, M. Victor Antony Raj, J. Madhavan, Der Chemica Sinica, 2, 158(2011).

34.A. E. Reed, R. B. Weinstock, F. Weinhold, J. Chem. Phys, 83, 735 - 746(1985). 
